\title{
The Step Feed Mechanisms For Auger Machines
}

\author{
Leonid Mametyev ${ }^{1}$, Oleg Lyubimov ${ }^{2, *}$, and Yuri Drozdenko ${ }^{1}$ \\ ${ }^{1}$ T.F. Gorbachev Kuzbass State Technical University, Department of mining machines and \\ complexes, 650000 Kemerovo, Vesennya str., 28, Russian Federation \\ ${ }^{2}$ T.F. Gorbachev Kuzbass State Technical University, Department of information and automated \\ manufacturing systems, 650000 Kemerovo, Vesennya str., 28, Russian Federation
}

\begin{abstract}
The paper presents the results of studies of technical solutions proposed in Kuzbass State Technical University and implemented in auger drilling equipment, characterized by the presence of a cyclic feed of a boring machine on the face and the corresponding reverse of the work by hydraulic jacks. Operation of complexes, which design feature is to provide a common plane for the axis of the boring hole and vectors of axial forces of the auger feeding mechanisms, was researched. The stress state of the cyclic step-feeding mechanism's functional elements by mathematical and software means of the finite element analysis is considered. The threshold values of the feed forces are determined from the point of view of ensuring the safety margin of welded joints used in the cyclic stepfeeding mechanism's structure, and from the point of operational reliability of the system as a whole. The obtained data allowed making recommendations for further improvement for the subsystem of the auger's cyclic step-feeding mechanism.
\end{abstract}

\section{Introduction}

Today, the optimization of energy consumption with simultaneous expansion of the range of bored holes for various purposes is a priority for the development of the horizontal boring (auger) equipment. At the same time, the two-stage technology has clear advantages. It involves boring a pioneer hole of small diameter at the first stage with an auger screw rod in a direct course and then boring it at the second stage to the desired diameter in a reverse course [1-8].

In terms of ensuring stability, those layout schemes of boring auger complexes have a particular perspective, whose design feature is to provide a common plane for the axis of the boring hole and vectors of axial forces of the auger feeding mechanisms [9-11].

The feeding drive can be implemented in two ways:

- using the reciprocating motion of the auger machine unit due to gear-rack gears, which is difficult to manufacture and reciprocal installation, sensitive to the presence of contamination and lack of lubrication;

\footnotetext{
* Corresponding author: oleglyub@gmail.com
} 
- using the cyclic feed of the auger machine unit on the face and the corresponding reverse of the work of hydraulic jacks, which is quite feasible in the light of the considered layout schemes $[12,13]$.

\section{Researches}

The preferred second method is demonstrated by technical solutions applied in auger complexes developed at KuzSTU [13-15]. The main structure includes a modular composite frame, a carriage with auger boring machine sliding along its guiding beams, a sectional casing string, an auger screw rod placed in it together with a tool for boring holes in direct or reverse courses. The feed of the carriage with the auger boring machine, associated with them auger screw rod in the casing string and the boring tool along guiding beams is provided by hydraulic jacks pivotally attached to it. Wherein, the movable stoppers 1 , rigidly attached to the hydraulic jack's stocks 2 , made with the possibility of engagement with the fixed stopper's 3 double-acting wedge surfaces. The dimensions are chosen such as the axes of the stocks 2 with the working strokes are in the same plane with the borehole axis. The movable stoppers 1 are kinematically integrated on the full-length stepping feed into the self-adjusting lifting or lowering devices 4 , which ensure locking engagement with either side of the fixed stoppers 3 on the frame during the working stroke of the carriage, with the simultaneous preventing engagement from its opposite side. In conditions of cyclic direct stepping feed, the design is under the influence of axial force $F_{c d s f}$; in conditions of cyclic reverse stepping feed $-F_{c r s}$; (Fig. 1).

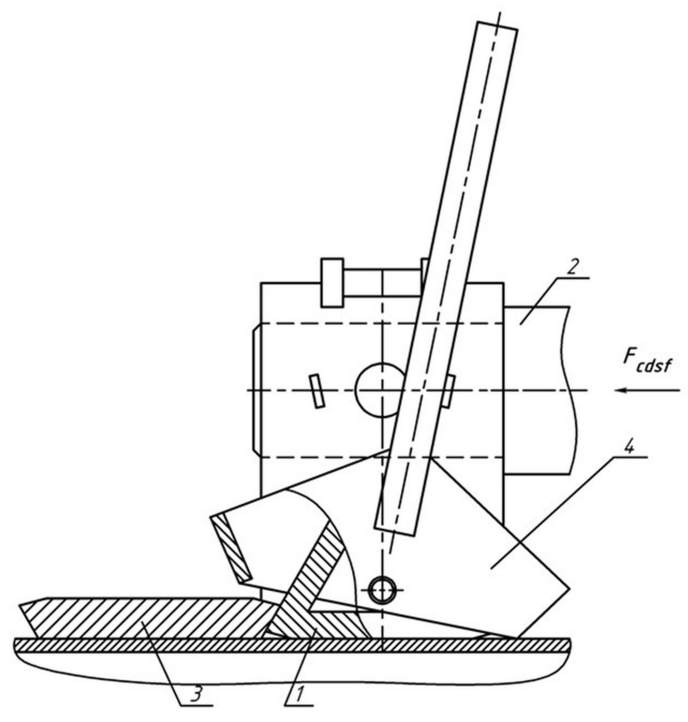

a

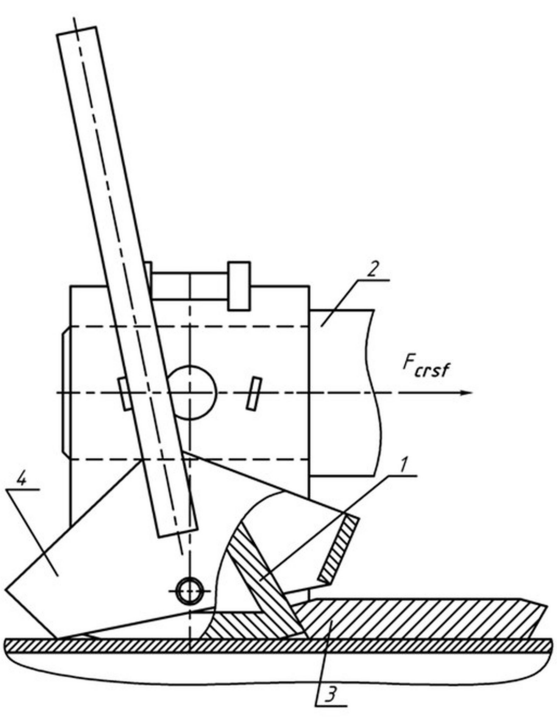

$\mathrm{b}$

Fig. 1. The engagement of movable and fixed stoppers: $a$ - for making a cyclic direct stepping feed; $b$ - for making a cyclic reverse stepping feed.

In the considerated structure, the operability of the cyclic step-feeding mechanisms depends primarily on the strength of the stoppers, the geometric characteristics of the sections and the physicomechanical properties of the materials of the parts of the lifting and lowering device. Consequently, there is a need for rapid assessment of their stress state under external loading conditions corresponding to the operating feed modes. 


\section{Results and discussion}

Using the mathematical facilities and software of the finite-element analysis, the change in the stress state of the movable stopper's structure was rated when the force of the hydraulic jack from 50 to $400 \mathrm{kN}$ was changed.

The threshold values of forces are established from the viewpoint on the ensuring safety factors of welded joints used in the movable stopper's structure for the self-adjusting lifting and lowering device. It is based on the results of the assessment of the revealed zones of increased normal stress in its elements (Fig. 2).

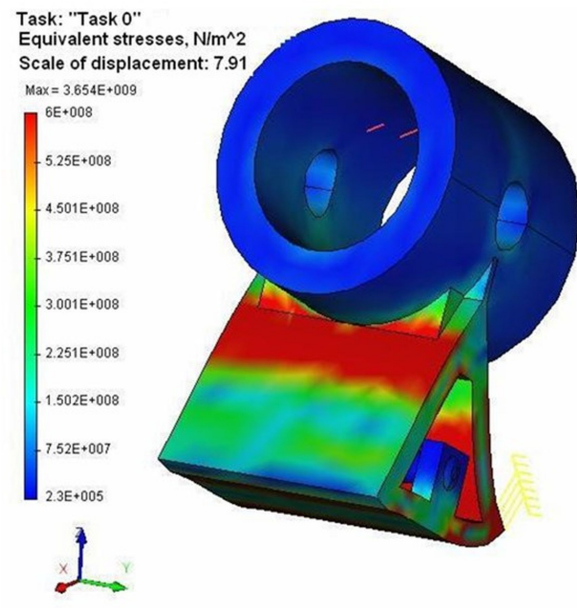

a

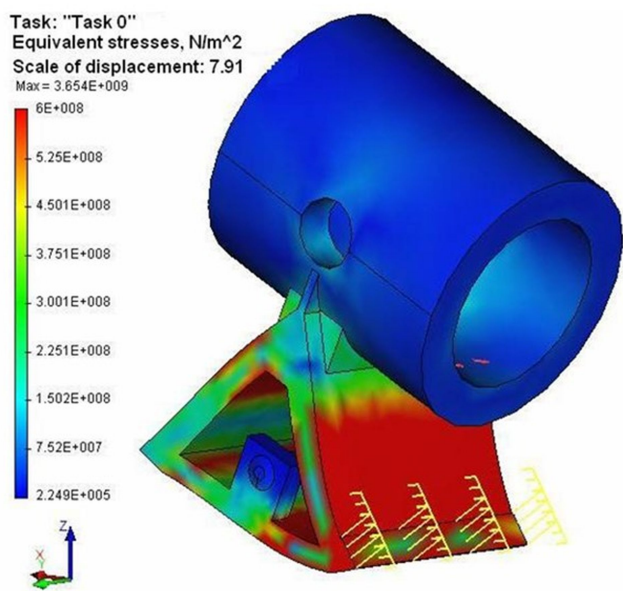

b

Fig. 2. The stress state of the movable stoper of the cyclic step-feeding mechanism at $F=200 \mathrm{kN}$ : a view from the side non-conjugated with a fixed stopper; $b$ - view from the side conjugated with a fixed stopper.

The stress concentrators in the functional elements of the initial version of the design of the step-feeding mechanism with the maximum level in the engagement places of the movable and fixed stoppers are revealed. They have the significant influence on the overall picture of the stress state of this subsystem of the cyclic step-feeding mechanism, and, consequently, the operational reliability of the system as a whole. Due to the significant size of the service zone, the design scheme requires taking into account the bending of the subsystem elements in the process of cyclic feed to direct or reverse moves.

The analysis of the obtained results made it possible to outline ways to further improvement the self-adjusting lifting and lowering device, which is proposed to be performed as a vertical support column 1 movably coupled to the stock 2 of the hydraulic jack 3 , and the turning in a vertical plane curved lever 4 pivotally attached to the cylinder of the hydraulic jack 3 with the possibility of raising or lowering the vertical support column 1 to exit or enter the locking engagement with the wedge sides of the fixed stoppers 5 of guiding beams 6 (Fig. 3).

Vertical support column 1 is made in the form of a double-ray prism with a central hole 7 , the surface of which is movably associated with the cylindrical surface of the stock 2 with the possibility of oriented rotation around its axis. Movable stoppers 8 are located at the ends of the rays opposite to each other relative to the longitudinal axis of the hydraulic jack 3, while one for locking engagement with a fixed stopper 5 on a direct course, and opposite for locking engagement with a fixed stopper 5 on a reverse course. 


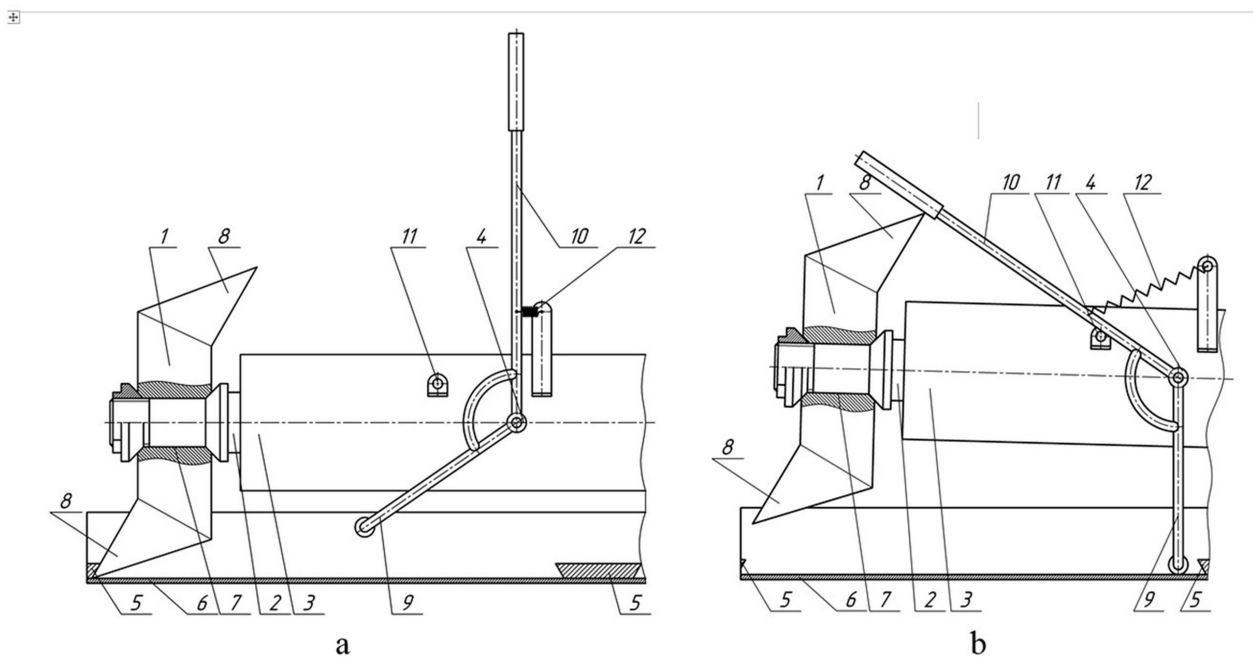

Fig. 3. The proposed structure for the lifting and lowering device: $a$ - initial position to adjust; $b$ working position to adjust.

A short executive arm 9 of the turning in a vertical plane curved lever 4 is made long enough to raise the vertical support column 1 to the working position or lower to the initial position to exit or enter the locking engagement with the wedge sides of the fixed stoppers 5 of the guiding beams 6, respectively. And it is placed in the original position in the gap between the cylinder of the hydraulic jack 3 and the upper surface of the fixed stoppers 5 of the guiding beams 6 , thus not preventing further operations. The long control arm 10 of the turning in a vertical plane curved lever 4 is provided with a rotation angle limiter 11 and an automatic return mechanism 12 .

The movable connection with the stocks 2 of the hydraulic jacks 3 allows each vertical support column 1 , lifted to the working position by the turning in the vertical plane curve lever 4 , to be tuned to a cyclical direct or reverse step-feeding movement. To do this, each vertical support column 1 , made in the form of a double-ray prism with a central hole 7 , the surface of which is movably associated with the cylindrical surface of the stock 2 , is rotated around its axis. The movable stoppers 8 are located at the ends of the rays opposite to each other relative to the longitudinal axis of the hydraulic jack 3 , while for subsequent locking engagement with a fixed stopper 5 on the direct course of the step-feeding mechanism, the corresponding movable stopper 8 takes the necessary position. Fixing of the vertical support column 1 in the form of a double-ray prism at the center hole 7, for example, can be carried out by means of a centering nut at the end of the stock 2 .

To facilitate the operation, the end of the short executive arm 9 may be provided with, for example, a roller.

The rotation angle limiter 11 of the curved lever 4 can be performed, for example, in the form of a bracket with a pin fixed on the cylinder of the hydraulic jack 3 . The automatic return mechanism 12 can be performed, for example, in the form of a bracket with a tension spring fixed on the cylinder of the hydraulic jack 3 (Fig 3).

Bringing after the adjustment and lowering to the initial position each of the vertical support column 1 by means of each of the turning in a vertical plane curved lever 4 allows to start the expansion or contraction of the stocks 2 .

The main advantage of the second variant of the step-feeding mechanism structure is, therefore, the separation in space of the high-speed elements of the lifting and lowering device. 


\section{Conclusion}

It has been established that the functional elements of the first variant of the step-feeding mechanism structure have stress concentrators with a maximum level in the conjugate zones of moving and fixed stoppers, which have significantly influence on the overall picture of the stress state of this cyclic feed mechanism subsystem and, consequently, on the whole system operational reliability.

The threshold values of the feed forces are determined from the point of view of ensuring the safety margin of welded joints used in the structure of the lifting and lowering device movable stopper. It is established that the design scheme requires taking into account the bending of the subsystem elements in the process of cyclic feed to the direct or reverse courses.

It is recommended to reduce the service zone to remove the control system of the cyclic feed mechanism from the stress state area in accordance with the second structure of the step-feeding mechanism, characterized by the presence of the spaced apart fast-acting elements of the lifting and lowering device.

\section{References}

1. M. Safokhin, L. Mamet'ev, A. Tsekhin, A. Anan'ev, J. of Min. Sc., 29, 4 (1993)

2. M. Safokhin, L. Mametev, V. Ivshin, O. Erin, N. Skornyakov, A. Ananev, Patent 2026977 of the Russian Federation

3. L. Mametyev, O. Lyubimov, 26th No-Dig Int. Conf. and Exh. (2008)

4. L. Mametyev, Yu. Drozdenko, O. Lyubimov, The 8th Rus.-Chin. Sym., 50 (2016)

5. L. Mamet'ev, A. Khoreshok, A. Tsekhin, A. Borisov, J. of Min. Sc., 51, 6 (2015)

6. A. Korotkov, V. Korotkov, L. Mametyev, L. Korotkova, T. Terjaeva, E3S Web of Conf., 15, 1503005 (2017)

7. L. Mamet'ev, O. Lyubimov, Yu. Drozdenko, J. of Min. Sc., 53, 2 (2017)

8. N. Kurishkin, O. Lyubimov, Ya. Mescheryakov, P. Oboyansky, E. Mametyev, K. Ponomaryov, EIIC-2013: Proc. in El. Intern. Interdisc. Conf., 401 (2013)

9. L. Mametyev, A. Khoreshok, A. Tsekhin, S. Mukhortikov, A. Borisov, The 8th Rus.Chin. Sym., 221 (2016)

10. L. Mamet'ev, A. Khoreshok, A. Tsekhin, A. Borisov, E3S Web of Conf., 15, 2103005 (2017)

11. L. Mametev, O. Ljubimov, Ju. Drozdenko, Patent 144475 of the Russian Federation

12. I. Bogomolov, M. Khusnutdinov, O. Ljubimov, D. Malishkin, Patent 2550703 of the Russian Federation

13. L. Mametev, O. Ljubimov, Ju. Drozdenko, E. Mametev, K. Ponomarev, Patent 156637 of the Russian Federation

14. L. Mametev, O. Ljubimov, Ju. Drozdenko, E. Mametev, K. Ponomarev, Patent 2578081 of the Russian Federation

15. L. Mametev, O. Ljubimov, Ju. Drozdenko, Patent 165050 of the Russian Federation

16. L. Mametev, O. Ljubimov, Ju. Drozdenko, V. Korobejnikov, Patent 185552 of the Russian Federation 